\title{
Effectiveness of tuberculosis prophylaxis in patients with HIV/AIDS - retrospective analysis of data from Almaty, Kazakhstan, 2010-2015
}

\author{
Konrad Juszkiewicz ${ }^{1, A-C, E \oplus \bullet}$, Mirosław J. Jarosz ${ }^{2, A, E \oplus}$, Anna Włoszczak-Szubzda ${ }^{2, C-F \oplus}$, \\ Magdalena Głowacka ${ }^{2, D} \oplus$ \\ ${ }^{1}$ KIT Royal Tropical Institute, Amsterdam, The Netherlands \\ ${ }^{2}$ University of Economics and Innovation, Lublin, Poland \\ A - Research concept and design, B - Collection and/or assembly of data, C - Data analysis and interpretation, \\ $D$ - Writing the article, E - Critical revision of the article, F - Final approval of article
}

Juszkiewicz K, Jarosz MJ, Włoszczak-Szubzda A, Głowacka M. Effectiveness of tuberculosis prophylaxis in patients with HIV/AIDS - retrospective analysis of data from Almaty, Kazakhstan, 2010-2015. Ann Agric Environ Med. 2020; 27(4): 695-701. doi: 10.26444/aaem/118611

\begin{abstract}
Introduction. According to data from the National Centre for Prevention and Control of AIDS, in the Republic of Kazakhstan, $45.8 \%$ of patients in the symptomatic stages of HIV infection are diagnosed with tuberculosis (TB) which is the cause of death in $36 \%$ of patients infected with HIV.

Objective. The aim of the study was to conduct a retrospective analysis of the effectiveness of tuberculosis (TB) chemoprophylaxis among people living with HIV in the Republic of Kazakhstan (RK) in Central Asia.

Materials and method. A retrospective analysis of patient health status was performed for each of the 648 patients (323 in the study group and 325 in the control group) during 2010-2015. Data from outpatient treatment charts were used concerning each patient infected with HIV observed at AIDS Treatment Centres. From among the 648 patients infected with HIV, 136 were receiving isoniazid in 2010, and 187 in 2011. The control group consisted of 325 people living with HIV (PLW HIV), who did not received isoniazid during observation.

Results. The incidence of TB in patients who underwent chemoprophylaxis did not exceed $0.555 / 100,000$ population in the first year of observation. Within 5 years, the TB incidence dropped to 0 . In the control group, the TB incidence rate during the first year of observation was 3.262/100,000, with a decrease to 0.364 observed in 2015 . Cumulated incidence rate in 2011-2015 in the study group accounted for 1.276/100,000. In the control group, the cumulative incidence was 4.3 times higher and accounted for 5.527. A significant difference in the mortality rate due to TB in the study and control groups was observed, the share of deaths due to TB in study group was $21.6 \%$ - nearly 3 times lower than in the control group (57.0\%). Conclusions. The effectiveness of chemoprophylaxis for TB depends on biomedical, organizational and cultural factors. The presence of HIV co-infections is a special situation. Opposite to the majority of reports, in own study, no drug-resistant forms of tuberculosis were observed in relation with chemoprophylaxis with isoniazid. In the examined population, TB chemoprophylaxis reduced the incidence and cumulative incidence of TB among PLW HIV by 3.4-4.8 times. Isoniazid chemoprophylaxis decreased 4-fold the annual and cumulative mortality due to TB.
\end{abstract}

- Key words

isoniazid, TB/HIV co-infection, chemoprophylaxis of TB, TB/HIV morbidity, TB/HIV

\section{INTRODUCTION}

In recent years, in the countries of Eastern Europe and Central Asia, morbidity due to tuberculosis among people infected with HIV has remained at a high level. Tuberculosis is also the leading opportunistic infection among people living with HIV (PLW HIV). According to data from the National Centre for Prevention and Control of AIDS, in the Republic of Kazakhstan (RK) tuberculosis is diagnosed in $45.8 \%$ of patients who are in the symptomatic stages of HIV infection, and is the cause of death in $36 \%$ of patients infected with HIV [1].

The situation is further complicated by the fact that diagnosis of tuberculosis in an HIV-infected individual is a very complicated medical task. In only $43-51 \%$ of cases are $M$. tuberculosis detected in sputum, and radiological

Address for correspondence: Magdalena Głowacka, University of Economics and Innovation, Lublin, Poland

E-mail: magdalenaglowacka1@gmail.com

Received: 15.02.2019; accepted: 02.03.2020; first published: 23.03.2020 changes in the lungs are often non-specific or atypical [2]. Therefore, many cases of non-diagnosed tuberculosis occur among patients infected with HIV. Approximately a half of the cases of tuberculosis in people living with HIV who were examined during the first year of observation after HIV diagnosis and exclusion of tuberculosis during the first dispensary examination.

Despite recommendations by the World Health Organization (WHO) concerning isoniazid prophylactic therapy (IPT) in order to prevent the development of an active form of tuberculosis in people living with HIV, the effectiveness of this therapy still creates doubts in many regional specialists concerned about the danger of the spread of drug-resistant tuberculosis in Central Asia. There is a simple reason for their logic, Kazakhstan is one of 30 countries prioritised by the WHO with a high-burden of multi drug-resistant (MDR)-Tuberculosis (TB). Moreover, an estimated $25 \%$ of all new TB cases have MDR-TB, while $43 \%$ of previously treated TB cases have MDR-TB. Considering this situation, and that the WHO proposes using isoniazid 
monotherapy, this approach could lead to an increase in multi-drug resistant cases $[3,4,5]$.

This study addresses one important question; is isoniazid preventive therapy effective for TB prophylaxis in patients living with HIV [6].

\section{OBJECTIVE}

The aim of the study was to conduct a retrospective analysis of the effectiveness of tuberculosis chemoprophylaxis among people living with HIV. The results could be used to develop recommendations for improving the quality of tuberculosis chemoprophylaxis, which should lead to a reduction in the number of tuberculosis cases, and reduce the mortality rate of people living with HIV in Kazakhstan.

\section{MATERIALS AND METHOD}

For the cross-sectional study, $648 \mathrm{HIV}$-positive patients from the dispensary records of the Centre for Prophylaxis and Treatment of AIDS in the city of Almaty were selected according to inclusion or exclusion criteria. From this patient population, 323 patients with HIV were selected. These patients had undergone isoniazid prophylactic treatment for 6 months (WHO recommended length of treatment) during 2010-2011 (136 patients received isoniazid in 2010; 187 patients received isoniazid in 2011). From the same group of 648 patients, 325 patients with HIV were selected as a control group. These patients did not receive IPT during 2010-2011.

A retrospective analysis of the health status was performed for each of the 648 patients (323 in the study group and 325 in the control group) during 2010-2015. Data from outpatient treatment charts were used for each patient infected with HIV observed at AIDS Treatment Centres.

The groups of patients who received isoniazid were analysed based on the following conditions:

- according to the clinical stage of the disease - I, II, III, IV;

- by gender - males, females;

- according to the risk group in terms of intravenous drug administration (IDU) (IDUs vs. non-IDU);

- according to the time of detection of HIV infection;

- new cases;

- remaining previously in the dispensary observation at the AIDS Centres.

During the study the following events were recoded and analysed:

1) chemoprophylaxis of TB after 2010-2011, time to start antiretroviral therapy (ARV);

2) pharmacological treatment according to the ARV scheme;

3) clinical stage of HIV infection;

4) T cell lymphocyte level indicator - helper with CD4 + receptors;

5) viral load by polymerase chain reaction (PCR);

6) examination of the level of HIV virus;

7) time to developing symptomatic tuberculosis;

8) costs of treatment;

9) time and cause of death (if it occurred).

In the study, the following inclusion criteria applied:

- PLW HIV, citizens of RK and residents of the city of
Almaty, entered in to the records at the AIDS Centre; - age over 18 years, patients who underwent a planned X-ray examination and patients with indications for chemo prophylaxis of TB.

The following patients were excluded from study:

- patients with active or undergoing tuberculosis treatment;

- pregnant women and women during the childbirth;

- patients under the age of 18 years;

- foreigners or anonymous clients.

In the study, the ratio of females to males in both the study group and the control group, was 1.5:1, while the ratio of patients' gender in the general dispensary group was 2.1:1.

The distribution of patients according to the clinical stage was the same in the study and control groups. The percentage of the clinical stage PLW HIV in the examined groups was slightly different from the distribution of PLW HIV, according to the clinical stage in the general dispensary group. In the examined groups, patients with clinical stage IV constituted more than $6 \%$, while in the general dispensary group - less than $3 \%$.

In the control group, similar calculations were made from the time of registration of PLW HIV into study as a new HIV case (in 2010), or from 31 December 2010, for all cases of HIV old infections, detected earlier than that year.

In general, the PLW HIV distribution according to the clinical stage was comparable in the study group and the general dispensary group. Differences in details were related to the quality of dispensarisation, planned dates of visits to the AIDS Centre, and to detailed examination with indications for performing TB chemoprophylaxis (Tab. 1).

In order to assess the outcomes of preventive treatment with isoniazid, in the study group and in the control group, the relative risk formula was used (RR). RR is the ratio between the probability of the occurrence of a given effect in the experimental group, in which a specified intervention was applied (e.g. surgical procedure or therapy with the examined drug), and its probability in the control group; this refers to cause-effect relationships, and describes the part of risk remaining after the intervention.

Statistical description of the data for quantitative variables included:

- treatment outcome numbers (n);

- $\operatorname{arithmetic~mean~}(\mathrm{M})$;

- standard deviation (SD).

Statistical description of the data for qualitative variables included:

- numbers;

- absolute frequency of each variable;

- relative frequency of each variable (percentage).

For all the investigated qualitative variables, the lack of data was not considered while calculating the relative frequency. The relationships between the discrete (qualitative) variables were verified using Pearson's chi-squared test. Statistical analysis of data was performed using the software IBM SPSS version 11.5 (SPSS Inc., Chicago, IL), and SAS version 9.2 (Cary, NC). 
Table 1. Comparative characteristics of the study group, control group and general disposable group - people living with HIV (PLW HIV), according to demographic, epidemiological and clinical indicators

\begin{tabular}{|c|c|c|c|c|c|c|c|}
\hline \multirow{2}{*}{ Indicators } & & \multicolumn{2}{|c|}{ Study group } & \multicolumn{2}{|c|}{ Control group } & \multicolumn{2}{|c|}{ General Dispensary Group (GDG) } \\
\hline & & $\mathbf{N}$ & $\%$ & $\mathbf{N}$ & $\%$ & $\mathbf{N}$ & $\%$ \\
\hline \multirow{4}{*}{ Gender } & Male & 192 & 59.4 & 193 & 59.4 & 1175 & 68.1 \\
\hline & Female & 131 & 40.6 & 132 & 40.6 & 551 & 31.9 \\
\hline & Total & 323 & 100.0 & 325 & 100.0 & 1.726 & 100.0 \\
\hline & Tests Chi ${ }^{2}$ & \multicolumn{6}{|c|}{ Study group vs. control group: $\mathrm{Chi}^{2}=0.0002 ; \mathrm{p}=0.987983$} \\
\hline \multirow{5}{*}{$\begin{array}{l}\text { General Dispensary } \\
\text { Group (GDG) }\end{array}$} & Male & 131 & 68.2 & 140 & 72.5 & 962 & 83.8 \\
\hline & Female & 43 & 32.8 & 41 & 31.1 & 186 & 16.2 \\
\hline & Total & 174 & 53.9 & 181 & 55.7 & 1148 & 100.0 \\
\hline & \multirow{2}{*}{ Tests Chi ${ }^{2}$} & \multicolumn{6}{|c|}{ Study group vs. control group: $\mathrm{Chi}^{2}=0.2086 ; \mathrm{p}=0.647895$} \\
\hline & & \multicolumn{6}{|c|}{ Study group vs. control group vs. general dispensary group: $\mathrm{Chi}^{2}=23.2743 ; \mathrm{p}<0.00001$} \\
\hline & II & 98 & 30.3 & 90 & 27.7 & 382 & 22.1 \\
\hline & III & 73 & 22.6 & 73 & 22.5 & 387 & 22.4 \\
\hline infection & IV & 20 & 6.2 & 21 & 6.5 & 48 & 2.8 \\
\hline & Total & 323 & 100.0 & 325 & 100.0 & 1726 & 100.0 \\
\hline & \multirow{2}{*}{ Tests Chi } & \multicolumn{6}{|c|}{ Study group vs. control group: $\mathrm{Chi}^{2}=0.6554 ; \mathrm{p}=0.883652$} \\
\hline & & Study group & group vs & pensa & $2=36.30$ & .00001 & \\
\hline \multirow{5}{*}{$\begin{array}{l}\text { Time to make a } \\
\text { diagnosis of 'HIV } \\
\text { infection' }\end{array}$} & New case & 118 & 36.5 & 127 & 39.1 & 252 & 14.6 \\
\hline & Previous detection & 205 & 63.5 & 198 & 60.9 & 1474 & 85.4 \\
\hline & Total & 323 & 100.0 & 325 & 100.0 & 1726 & 100.0 \\
\hline & \multirow{2}{*}{ Tests Chi ${ }^{2}$} & \multicolumn{6}{|c|}{ Study group vs. control group: $\mathrm{Chi}^{2}=0.446 ; \mathrm{p}=0.504225$} \\
\hline & & \multicolumn{6}{|c|}{ Study group vs. control group vs. all: $\mathrm{Chi}^{2}=153.9423 ; \mathrm{p}<0.00001$} \\
\hline
\end{tabular}

Source: Own study based on data from the 'Electronic Registry and Monitoring of HIV Infections Cases in RK (Republic of Kazakhstan)'.

\section{RESULTS}

The incidence of $\mathrm{TB}$ in patients who underwent chemoprophylaxis did not exceed $0.555 / 100,000$ population in the first year of observation. Within 5 years, the incidence of TB dropped to zero. TB incidence rate in the study group was clearly lower when compared with the control group (Fig. 1). In the control group, the TB incidence rate during the first year of observation was 3.262/100,000, with a decrease to 0.364 observed in 2015 (fifth year of study).

One of the most important research indicators in the study group was the time of TB disease diagnosis, calculated from the end of the isoniazid chemoprophylaxis cycle to the diagnosis of active TB in patients with HIV. After TB chemoprophylaxis,
21 cases of active tuberculosis were recorded, an average of $19.9 \pm 11.95$ months from the end of the prophylactic treatment cycle. In the control group who did not receive TB chemoprophylaxis, the mean active $\mathrm{TB}$ registration time in 91 patients - Mean value $(M)=22.4$, with Standard Deviation $(\mathrm{SD})= \pm 15.09$ months. $51.6 \%$ of cases of active TB in patients who did not receive chemoprophylaxis with isoniazid were already registered in the first year of observation (Tab. 2).

Cumulated incidence rate in 2011-2015 in the study group accounted for 1.276 per 100,000 . In the control group, the cumulative incidence was higher by 4.3 times and accounted for 5.527. According to the data from the National Centre for Tuberculosis, TB/ HIV incidence in 2014 is 3.4 cases per 100,000 population (Fig. 2).

Table 2. Registered cases of active TB and incidence of TB among patients in the study group and control group during 2011 - 2015 (absolute numbers and \%)

\begin{tabular}{|c|c|c|c|c|c|c|}
\hline \multirow{2}{*}{ PLW HIV groups } & & \multicolumn{5}{|c|}{ N0. of cases of active TB during the period of observation } \\
\hline & & 2011 & 2012 & 2013 & 2014 & 2015 \\
\hline \multirow{3}{*}{ Study group, $n=323$} & Numbers & 8 & 6 & 4 & 3 & 0 \\
\hline & $\%$ of PLW HIV group; $n=323$ & 2.5 & 1.9 & 1.2 & 0.9 & 0 \\
\hline & $\%$ of number of TB $n=21$ cases & 38.1 & 28.6 & 19.0 & 14.3 & 0 \\
\hline \multicolumn{2}{|c|}{ Incidence of TB per 100,000 inhabitants in the study group } & 0.555 & 0.398 & 0.254 & 0.187 & 0 \\
\hline Control group, $\mathrm{n}=325$ & $\%$ of PLW HIV group; $n=325$ & 14.5 & 5.2 & 3.1 & 3.4 & 1.8 \\
\hline & $\%$ of number of TB; $n=91$ & 51.6 & 18.7 & 11.0 & 12.1 & 6.6 \\
\hline \multicolumn{2}{|c|}{ Incidence of TB per100,000 in the control group } & 3.262 & 1.126 & 0.634 & 0.685 & 0.364 \\
\hline
\end{tabular}

Source: Own study based on the 'Electronic Register and Monitoring of Cases of HIV infection in RK (Republic of Kazakhstan)'. 


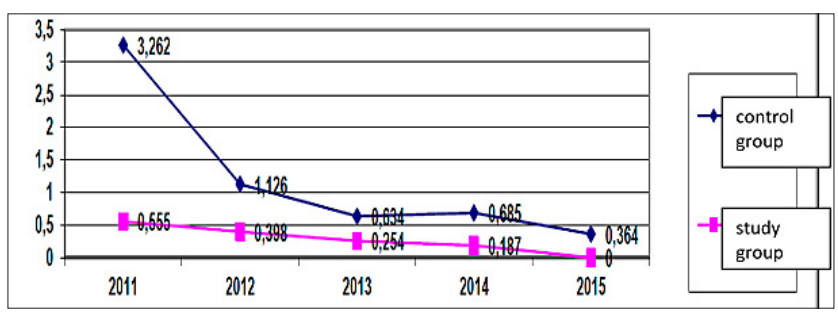

Figure 1. Incidence rate of TB in the study group and control group in 2011-2015. Source: Own study based on 'Electronic Register and Monitoring of Cases of HIV infection in RK (Republic of Kazakhstan)'

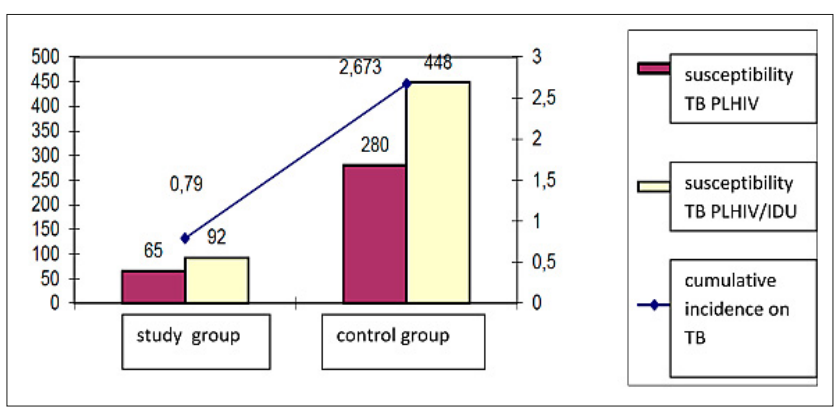

Figure 2. Level of susceptibility to TB among PLW HIV (people living with HIV) and PLW HIV / IDU (intravenous drug users) and cumulative incidence rate of TB per 100,000 population in $2011-2015$.

Source: Own study based on 'Electronic Register and Monitoring of Cases of HIV infection in RK (Republic of Kazakhstan)'

During the first 12 months after completion of the TB chemoprophylaxis cycle, a substantial difference in the number of active tuberculosis cases was observed between the study and control groups. Among patients who did not receive isoniazid prophylactic treatment, active TB was registered 3.4 times more often than among PLW HIV who underwent chemoprophylaxis. Within 12-60 months, in the group of patients who received isoniazid, active tuberculosis was registered 5.8 less often than in the PLHIV group, which did not participate in prophylactic therapy.

Cumulative incidence of TB in the years 2012-2015 in the study group was 0.790 cases per 100,000 population. During the same period in the control group, cumulative morbidity due to TB was 3.4 times higher 2.673 cases per 100,000 . Within 60 months, TB in the study group was significantly lower than in the control group; with $93.3 \%$ of treated patients not suffering from tuberculosis compared to the $69.5 \%$ in the control group (Death Incidence DI 0.12-0.60; $\mathrm{p}<0.0008$ ) (Fig. 3).

A significant difference in the mortality rate due to $\mathrm{TB}$ was observed between the study and control groups. The

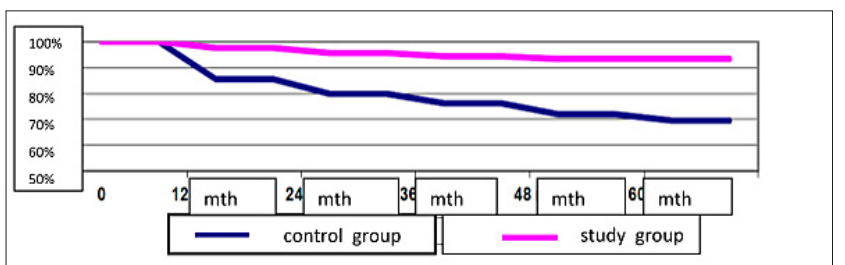

Figure 3. Percentage of patients with HIV infection without symptoms of active tuberculosis in the study group and in the control group within 60 months. Source: Own study based on 'Electronic Register and Monitoring of Cases of HIV infection in RK (Republic of Kazakhstan)'

share of deaths due to TB among patients who underwent the TB chemoprophylaxis cycle was $21.6 \%$, which was almost 3 times lower than in the control group (57.0\%) who did not receive isoniazid $(\mathrm{p}=0.020972)$. Registered mortality was also twice as low than that in the general group (44.2\%), although this relationship was not statistically significant $(\mathrm{p}=0.199297)$ (Tab. 3).

Causes of death due to other types of diseases in both groups did not show any statistical differences. Mortality due to tuberculosis was almost the same in both groups - 381 per 1,000 TB/HIV cases in the study group and 363 in the control group. The percentage of patients surviving after 5 years of observation in the study group was $88.5 \%$, while in the control group $-82.2 \%$ (Fig. 4).

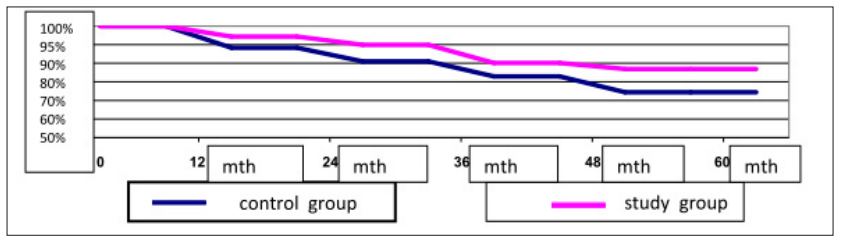

Figure 4. Percentage of living patients with HIV infection in the study and control groups.

Source: Own study based on 'Electronic Register and Monitoring of Cases of HIV infection in RK (Republic of Kazakhstan)'

It is noteworthy that in recent years the susceptibility of females to HIV infection and a higher risk of the disease has led to an increase in the percentage of females in the general group. At the end of 2015, the ratio of females to males in this group was 1.5:1. The higher number of females in the examined groups was due to a higher percentage of new cases of HIV infection among them, as well as a lower detection in women of active pulmonary tuberculosis during radiologic examinations (Fig. 5).

Table 3. Main causes of deaths of patients in the study. control and general dispensary groups 2011-2015

\begin{tabular}{|c|c|c|c|c|c|c|}
\hline \multirow{2}{*}{ Causes of death } & \multicolumn{2}{|c|}{ Study Group } & \multicolumn{2}{|c|}{ Control Group } & \multicolumn{2}{|c|}{ General Dispensary Group } \\
\hline & $\mathrm{N}$ & $\%$ & $\mathrm{~N}$ & $\%$ & $\mathrm{~N}$ & $\%$ \\
\hline Tuberculosis & 8 & 21.6 & 33 & 57.0 & 221 & 44.2 \\
\hline Infectious diseases, incl. pneumonia & 10 & 27.0 & 8 & 13.8 & 89 & 17.8 \\
\hline Inflammation and cirrhosis of the liver & 6 & 16.2 & 5 & 8.6 & 70 & 14.0 \\
\hline Oncological diseases & 7 & 18.9 & 6 & 10.3 & 64 & 12.8 \\
\hline Total & 37 & 100.0 & 58 & 100.0 & 500 & 100.0 \\
\hline \multirow{2}{*}{ Tests $\mathrm{Chi}^{2}$} & \multicolumn{6}{|c|}{ Study group vs. control group: $\mathrm{Chi}^{2}=11.5566 ; \mathrm{p}=0.020972$} \\
\hline & \multicolumn{6}{|c|}{ Study group vs. general group: $\mathrm{Chi}^{2}=7.5553 ; \mathrm{p}=0.109297$} \\
\hline
\end{tabular}




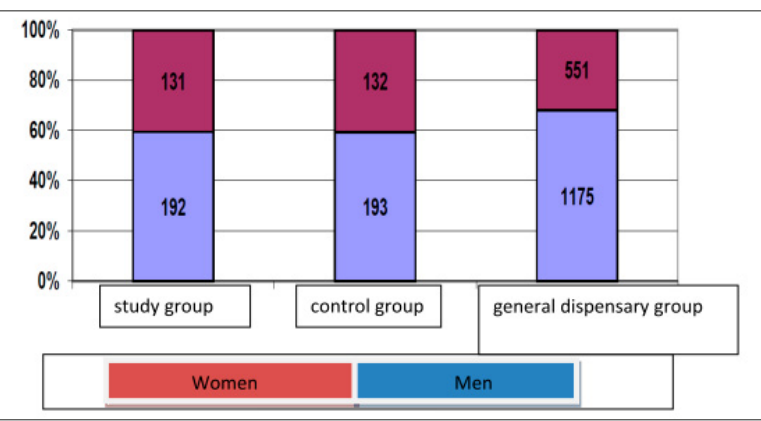

Figure 5. Gender structure in the study group and the general group (absolute numbers in the percentage scale).

Source: Own study based on 'Electronic Register and Monitoring of Cases of HIV infection in RK (Republic of Kazakhstan)

Intravenous drug users (IDU) constituted an important group at risk of tuberculosis. In the general group, the IDU constituted $66.5 \%$ of all patients, whereas among all patients registered in outpatient dispensaries, IDU females accounted for about 16.2\% (186), while males $83.8 \%$ (962) (Fig. 6).

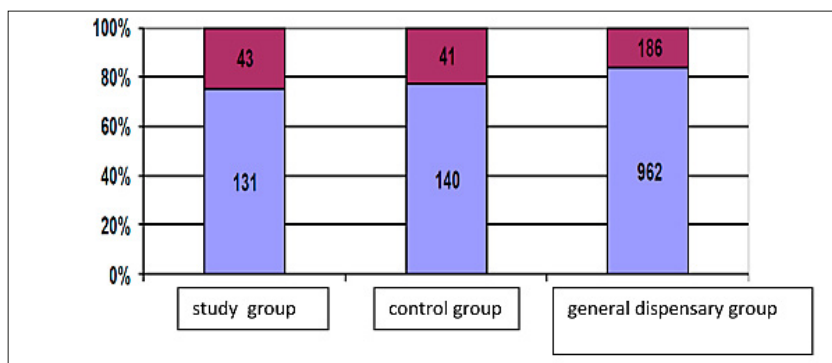

Figure 6. Intravenous drug users (IDU) in the study, control and general groups by gender (absolute numbers in the percentage scale).

Source: Own study based on 'Electronic Register and Monitoring of Cases of HIV infection in RK (Republic of Kazakhstan)

From among all the patients qualified for the study $(648$ patients: study group + control group) the percentage of IDU was $54.8 \%$. The ratio of PLW HIV females to males using intravenous drugs was 3:1.2. In turn, in the general group, this ratio was 5:1, female to male. However, in the study group and the control group, the ratio of both IDU males and females was practically the same. The higher percentage of IDU in the general group, compared to the study group, is justifies by a high frequency of occurrence of active tuberculosis among IDU males.

\section{DISCUSSION}

Results of international studies concerning analysis of the scope of problems similar to those undertaken in own study in patients with tuberculosis and HIV, in many cases differed from those obtained in own study. Studies conducted in Kampala, Uganda (Johnson JL. et al.), demonstrated that 6-month administration of isoniazid provided a short-term protection against tuberculosis, whereas 3-month regimens of isoniazid plus rifampicin, or isoniazid puls, rifampicin and pyrazinamide, provided permanent protection for up to 3 years [7]. A study conducted in Zambia (Quigley MA et al.), showed that HIV patients infected with tuberculosis who were administered isoniazid for 6 months, and rifampicin and pyrazinamide for 3 months or placebo, were similarly protected against tuberculosis - approx. 2.5 years [8].

In both cases, the protection against tuberculosis was shorter than in own study where, based on the WHO recommendations, a 6-month regimen of treatment with isoniazid was applied.

Studies conducted concerning this problem do not equivocally show which method of administration of isoniazid is best in considering time. Directly observed 3-month administration once a week of isoniazid plus rifampicin, was at least as effective as a 9-month daily administration of isoniazid, mainly in patients not infected with HIV, and living in countries with low or mediocre morbidity due to tuberculosis [9]. Own study indicated that a centralised time of administration of isoniazid (6 months) was very effective. Chemoprophylaxis for TB reduced the occurrence of tuberculosis among people living with HIV, and the cumulative incidence of tuberculosis by 3.4-4.8 times.

A study conducted in Botswana (Samandari T et al.) in patients with tuberculosis infected with HIV, who were administered 6 months' open-label isoniazid, and subsequently 30 -months masked placebo (control group), or administration of 6 months' open-label isoniazid, and subsequently 30-months masked isoniazid, showed that in tuberculosis endemic conditions, a 36-month isoniazid prophylaxis was more effective in the prevention of tuberculosis than 6-month prophylaxis in people living with HIV, and above all, it benefited those who had a positive tuberculin skin test [10]. In India, (Swaminathan S et al.) an open randomized clinical study was conducted in patients infected with HIV, who had not previously undergone antiretroviral therapy, and without active TB. These patients were randomly classified into a group receiving ethambutol at a dose of $800 \mathrm{mg}$ and isoniazid at a dose $300 \mathrm{mg}$ daily for 6 months $(6 \mathrm{EH})$, or isoniazid at a dose of $300 \mathrm{mg}$ daily for 36 months $(36 \mathrm{H})$. According to the researchers, both therapies were equally effective in the prevention of tuberculosis, compared to historical morbidity rates. However, a tendency was observed towards a decrease in the incidence of tuberculosis at $36 \mathrm{H}$. No increase was observed in resistance to isoniazid, compared to the expected indicator in patients infected with HIV [11].

The above-mentioned differences between studies were mostly related to different approaches to the problem, different methodology, often associated with the country of study or selection of patients for observation. Not all studies concerned exclusively prophylaxis with isoniazid for patients living with HIV infected with tuberculosis $[12,13]$. In turn, in the review by British researchers (Balcells ME et al.), pertaining to studies of the effectiveness of IPT, in 5 electronic databases (PubMed, Embase, Popline, National Library of Medicine Gateway, Cochrane Library) from which 13 reports were selected defining the problem in similar way, a conclusion was drawn similar to own study, that IPT is a safe, cheap intervention, which may potentially reduce the number of diseases and deaths due to tuberculosis, especially in people living with HIV. The main cause of resistance to anti-tuberculosis drugs is an improper treatment of active tuberculosis. Therefore, any risk of a slight increase in the frequency of occurrence of resistance to isoniazid related with a wide use of IPT should be compared to the benefits related with a decrease in the incidence of tuberculosis [14]. 
The common denominator of the majority of studies or projects was the fact that in the analysis of preventive treatment the researchers used national or international databases. In all studies, isoniazid was a generally safe and well-tolerated drug, without significant side-effects. A good example may be the literature review conducted by researchers (Briggs MA et al.) from the Centers for Disease Control and Prevention, Atlanta, GA, entitled 'Use of Isoniazid Preventive Therapy for Tuberculosis Prophylaxis Among People Living with HIV/AIDS'. Reports were analyzed from the years 1995-2013 (41 articles), which allowed the conclusion that the WHO recommendations concerning the use of IPT for PLW HIV in order to reduce morbidity due to tuberculosis serve to emphasize the strengthening of the need for introduction of IPT [15]. Similar meta-analysis was performed by an international team of researchers from Ethiopia and Holland (Ayele HTetal.). A comprehensive search of PubMed, Excerpta Medica dataBASE (EMBASE), the Cochrane Central Register of Controlled Trials (CENTRAL) and Cumulative Index of Nursing and Allied Health (CINAHL) was performed to identify all relevant studies in the English language available from the start of MEDLINE to 11 September 2015. This meta-analysis suggests the protective effect of IPT on the development of tuberculosis in patients infected with HIV, patients with a positive result of tuberculin skin test (TST), benefit to a greater degree than those with negative TST. The effect on mortality and advancement of HIV was rather small; however, there is some evidence indicating an additional benefit from IPT, compared to anti-retroviral therapy (ART) only, in the prevention of tuberculosis [16].

Answers to the questions 'is prophylaxis using isoniazid effective?', and 'to what extent it results in a decrease in mortality and incidence of tuberculosis infection in patients with HIV?', are similar in international and Polish studies. Nevertheless, some differences occurred in the results of studies, most probably related with the level of spreading of tuberculosis and HIV, as well as economic situation and level of medical services in a given country or region. The Republic of Kazakhstan belongs to countries with a very high percentage of tuberculosis cases with bacteria resistant to isoniazid and rifampicin (more than 20\%) [5].

A subsequent study conducted in Ethiopia (Semu M et al.), also confirmed the thesis posed in own study. According to the authors of this report, IPT considerably reduced the incidence of tuberculosis (by $96.3 \%$ ), compared to patients who did not receive IPT. In researchers' opinion, because IPT considerably prevented the occurrence of active tuberculosis for 3 years, it should be strengthened even more in countries at high risk of TB-HIV [17]. Similar conclusions were also dawn by researchers from the Department of Medical Laboratory Sciences, College of Medicine and Health Sciences, Arba Minch University, Arba Minch, Ethiopia (Abossie A. et al.). The results of their study demonstrated that IPT considerably contributed to the reduction of tuberculosis load in patients with ART, more than in the group without IPT. This result emphasized the use of IPT in the prevention of tuberculosis, especially in all patients with ART [18].

The effectiveness of IPT is also confirmed by a study conducted in the Centre for the AIDS Programme of Research in in Durban, South Africa (CAPRISA). The researchers (Maharaj B. et al.), based on the results, clearly stated that IPT implemented in patients with tuberculosis who had been previously receiving ART, was well tolerated, with a good percentage of treatment completions, and a smaller number of TB cases diagnosed after application of IPT [19].

The key element of tuberculosis prophylaxis among the PLW HIV is the development and implementation of modern approaches to the rapid diagnosis of tuberculosis in various models of care, and wider support of health care systems in this respect. Progress in this area is currently noticeable.

Researchers from the Data Management, Statistics, and Evaluation Branch, Division of Tuberculosis Elimination, National Center for HIV/AIDS, Viral Hepatitis, STD, and TB Prevention, Atlanta, Georgia (Njie GJ. et al.), analyzed relevant literature from 2006-2017 in electronic databases (MEDLINE, Embase, CINAHL, Cochrane Database Library, Scopus and Clinicaltrials.gov.), observed that even a 3-month regimen of treatment with isoniazid and rifampicin is equally as safe and effective as other regimens of treatment of latent tuberculosis recommended by the WHO, and higher treatment completion rates are achieved [20].

\section{CONCLUSIONS}

The effectiveness of chemoprophylaxis for TB depends on many biomedical, organizational and cultural variables, which is confirmed by the results of studies conducted in many countries which differ by epidemiological and social situation, as well as the state of the health care system. The scale of HIV co-infections is a special situation.

The majority of reports demonstrate that chemoprophylaxis for $\mathrm{TB}$ reduced the primary incidence of tuberculosis. However, contrary to these studies, in own study no results were observed which would indicate the occurrence of drug-resistant forms of tuberculosis due to ioniazid chemoprophylaxis, and the results obtained allow the following conclusions with respect to the examined population:

1) Chemoprophylaxis for TB reduced the primary incidence of tuberculosis.

2) Chemoprophylaxis for TB reduced the occurrence of tuberculosis among people living with HIV, and the cumulative incidence of tuberculosis by 3.4-4.8 times;

3) Within 60 months, the delayed effectiveness of TB chemoprophylaxis performed among people living with HIV was $23.8 \%$ higher compared to the control group.

4) The performance of TB chemoprophylaxis decreased 4 -fold the annual and cumulative mortality due to tuberculosis.

5) The occurrence of TB among people living with HIV who were intravenous drug users was 1.4-4 times higher than in the general population of people living with HIV.

6) The incidence of tuberculosis in the group receiving isoniazid and in the group not receiving chemoprophylaxis of TB was 5 times higher than in non-injecting drug users.

\section{Abbreviations}

AIDS - Acquired Immune Deficiency Syndrome

HIV - Human Immunodeficiency Virus

TB - tuberculosis

RK - Republic of Kazakhstan

PLW HIV - people living with HIV

WHO - World Health Organization

IPT - Isoniazid prophylactic therapy

MDR - multi drug-resistant

IDU - injection drug user 
ARV - anti-retroviral (drug)

CD4 - cluster of differentiation 4

$\mathrm{PCR}$ - polymerase chain reaction

$\mathrm{RR}$ - relative risk

$\mathrm{n}$ - numbers

$\mathrm{M}$ - arithmetic mean

SD - standard deviation

$6 \mathrm{EH}$ - Isoniazid $(\mathrm{H})$ combination for 6 months

$36 \mathrm{H}$ - Isoniazid $(\mathrm{H})$ combination for 36 months

TST - tuberculin skin test

ART. - anti-retroviral therapy

STD - sexually transmitted diseases

\section{REFERENCES}

1. WHO. Global Tuberculosis control. Geneva 2018. http://apps.who.int/ iris/bitstream/handle/10665/274453/9789241565646-eng.pdf?ua=1 (access: 20-29.11.2018)

2. Padmapriyadarsini C, Narendran G, Swaminathan S. Diagnosis \& treatment of tubercu-losis in HIV co-infected patients. Indian J Med Res. 2011; 134(6): 850-865.

3. Stagg HR, Lipman MC, McHugh TD, Jenkins HE, Isoniazid resistant tuberculosis - a cause for concern? Int J Tuberc Lung Dis. 2017; 1; 21(2): 129-139.

4. WHO - Recommendation on 36 months isoniazid preventive therapy to adults and ado-lescents living with HIV in resource-constrained and high TB and HIV-prevalence set-tings: 2015 update. (access: 20-29.11.2018)

5. KIT - Royal Tropical Institute project: Addressing Drug-Resistant Tuberculosis in Kazakhstan. https://www.kit.nl/project/addressingdrug-resistant-tuberculosis-in-kazakhstan/ (access: 24.06.2019).

6. Juszkiewicz K, Jarosz MJ, Włoszczak-Szubzda A, Głowacka M. Comparative analysis of the effectiveness of tuberculosis prophylaxis in patients with HIV/AIDS treated with isoniazid. Ann Agric Environ Med. 2019; 26(3): 462-467.

7. Johnson JL, Okwera A, Hom DL, Mayanja H, MutuluuzaKityo C, Nsubuga P, et al. Duration of efficacy of treatment of latent tuberculosis infection in HIV-infected adults. AIDS. 2001; 15(16): 2137-2147.

8. Quigley MA, Mwinga A, Hosp M, Lisse I, Fuchs D, Porter JDH, et al. Long-term effect of preventive therapy for tuberculosis in a cohort of HIV-infected Zambian adults. AIDS. 2001; 15(2): 215-222.
9. Person AK, Sterling TR. Treatment of Latent Tuberculosis Infection in HIV: Shorter or Longer? Curr HIV/AIDS Rep. 2012; 9(3): 259-266.

10. Samandari T, Agizew TB, Nyirenda S, Tedla Z, Sibanda T, Shang N, Mosimaneotsile B, Motsamai OI, Bozeman L, Davis MK, Talbot EA, Moeti TL, Moffat HJ, Kilmarx PH, Castro KG, Wells CD. 6-month versus 36-month isoniazid preventive treatment for tuberculosis in adults with HIV infection in Botswana: a randomised, double-blind, placebo-controlled trial. Lancet. 2011; 377(9777): 1588-98.

11. Swaminathan S, Menon PA, Gopalan N, Perumal V, Santhanakrishnan RK, Ramachandran R, Chinnaiyan P, Iliayas S, Chandrasekaran P, Navaneethapandian PD, Elangovan T, Pho MT, Ware F, Paranji Ramaiyengar N. Efficacy of a six-month versus a 36-month regimen for prevention of tuberculosis in HIV-infected persons in India: a randomized clinical trial. PLoS One. 2012; 7(12): e47400.

12. Getahun H, Granich R, Sculier D, Gunneberg C, Blanc L, Nunn P, Raviglione M. Bar-riers and solutions, AIDS. 2010; 24 (suppl 5): S57S65.

13. Akolo C, Adetifa I, Shepperd S, Volmink J. Treatment of latent tuberculosis infection in HIV infected persons. Cochrane Database Syst Rev. 2010; 1(CD000171)

14. Balcells ME, Thomas SL, Godfrey-Faussett P, Grant AD. Isoniazid preventive therapy and risk for resistant tuberculosis. Emerg Infect Dis. 2006; 12: 744-751.

15. Briggs MA, Emerson C, Modi S, Taylor NK, Date A., Use of isoniazid preventive therapy for tuberculosis prophylaxis among people living with HIV/AIDS: a review of the literature. J Acquir Immune Defic Syndr. 2015; 68 (Suppl3): S297-305.

16. Ayele HT, Mourik MS, Debray TP, Bonten MJ. Isoniazid Prophylactic Therapy for the Prevention of Tuberculosis in HIV Infected Adults: A Systematic Review and Meta-Analysis of Randomized Trials. PLoS One. 2015; 9; 10(11): e0142290.

17. Semu M, Fenta TG, Medhin G, Assefa D. Effectiveness of isoniazid preventative therapy in reducing incidence of active tuberculosis among people living withHIV/AIDS in public health facilities of Addis Ababa, Ethiopia: a historical cohort study. BMC Infect Dis. 2017; 3; 17(1): 5.

18. Abossie A, Yohanes T. Assessment of isoniazid preventive therapy in the reduction of tuberculosis among ART patients in Arba Minch Hospital, Ethiopia. Ther Clin Risk Manag. 2017; 13: 361-366.

19. Maharaj B, Gengiah TN, Yende-Zuma N, Gengiah S, Naidoo A, Naidoo K. Imple-menting Isoniazid Preventive Therapy in a TB-treatment experienced cohort on ART. Int J Tuberc Lung Dis. 2017; 01; 21(5): 537-543.

20. Njie GJ, Morris SB, Woodruff RY, Moro RN, Vernon AA, Borisov AS. Isoniazid-Rifapentine for Latent Tuberculosis Infection: A Systematic Review and Meta-analysis. Am J Prev Med. 2018; 55(2): 244-252. 